\title{
P43 The Association of Irritable Affective Temperament with 24-Hour Brachial and Central Blood Pressure and Hemodynamic Parameters in Untreated Hypertensive Men
}

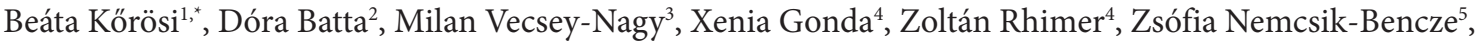
Andrea László ${ }^{6}$, János Nemcsik ${ }^{7}$

${ }^{1}$ Semmelweis University, Budapest, Hungary

${ }^{2}$ Department of Family Medicine, Semmelweis University, Budapest, Hungary

${ }^{3}$ MTA-SE Cardiovascular Imaging Research Group, Semmelweis University, Budapest, Hungary

${ }^{4}$ Department of Psychiatry and Psychotherapy, Semmelweis University, Budapest, Hungary

${ }^{5}$ Magnetic Resonance Research Center, Semmelweis University, Budapest, Hungary

${ }^{6}$ First German Hospital for Traditional Chinese Medicine, Bad Kötzting, Germany

${ }^{7}$ Health Service of Zugló (ZESZ), Budapest, Hungary

\begin{abstract}
Background: Affective temperaments (depressive, irritable, hyperthymic, anxious, cyclothymic) have important role in psychopathology, but cumulating data support their involvement in vascular pathology, especially in hypertension as well. The aim of our study was to evaluate their associations with 24-hour brachial and central hemodynamic parameters in untreated patients who were studied because of elevated office blood pressure.

Methods: Mobil-O-Graph was used to measure the 24-hour brachial and central parameters. Affective temperaments, depression and anxiety were evaluated with TEMPS-A, Beck and HAMA questionnaires, respectively.

Results: 84 patients were involved into the study (49 men). Irritable temperament score was associated with nighttime brachial systolic blood pressure $(r=0.279, p=0.010)$, with nighttime brachial mean arterial pressure $(r=0.239, p=0.028)$, with nighttime central systolic blood pressure $(r=0.288, p=0.008)$, with nighttime central diastolic blood pressure $(r=0.238, p=0.029)$, with 24-hour cardiac output $(r=0.298, p=0.006)$ and inversely with 24-hour reflection magnitude $(r=-0.297, p=0.006)$. These associations were attenuated after the adjustment of age, depression and anxiety and were absent in women.

Conclusion: In conclusion, affective temperaments, especially irritable temperament can have an impact on 24-hour brachial and central hemodynamics in untreated hypertensive men.

(c) 2019 Association for Research into Arterial Structure and Physiology. Publishing services by Atlantis Press International B.V. This is an open access article distributed under the CC BY-NC 4.0 license (http://creativecommons.org/licenses/by-nc/4.0/).
\end{abstract}

